\title{
Efeitos da Educação Física nos níveis de aptidão cardiorrespiratória e no índice de massa corporal na Educação de Jovens e Adultos - EJA
}

http://dx.doi.org/10.11606/1807-5509201900040639

\author{
Vanilson Batista LEMES* \\ Caroline BRAND* \\ Rodrigo Baptista MOREIRA** \\ Adroaldo Cezar Araujo GAYA* \\ Anelise Reis GAYA*
}

*Universidade Federal do Rio Grande do Sul, Porto Alegre, RS, Brasil.

**Universidade Luterana do Brasil, São Jerônimo, RS, Brasil.

\section{Resumo}

0 objetivo do presente estudo foi verificar o efeito das aulas de educação física escolar (EFI) nos níveis de aptidão cardiorrespiratória e no índice de massa corporal (IMC) em estudantes na Educação de Jovens e Adultos (EJA). Trata-se de um estudo de caso, avaliativo somativo realizado em uma escola do RS, elaborado com 8 sujeitos dos 16 aos 52 anos de ambos os sexos, de uma população total de 74, os quais cumpriram 75\% de frequência nas aulas de EFI na EJA. A aptidão cardiorrespiratória (APCR) e o índice de massa corporal (IMC), foram avaliados através da bateria de testes PROESP-Br no início do primeiro semestre do ano de 2015 e ao final desse periodo. Os efeitos da EFI no grupo foram mensurados a partir do modelo estatístico de equações generalizadas (GEE) em intervalos de confiança de 95\% (IC95\%) e delta médio percentual. Também foram descritos os percentuais de variação da APCR e do IMC para cada sujeito. Desse modo, verificou-se que a APCR aumentou significativamente do pré-teste $(675,25$ metros) para o pós-teste $(735,13$ metros), (IC95\% $=+17,2$ a $+102,55)$ o que corresponde a um efeito médio de $8,8 \%(p=0,006)$. Todos os sujeitos aumentaram a APCR na avaliação individual, tais resultados não foram observados no IMC. Concluímos que programa de educação física escolar contribuiu para a melhora dos niveis de aptidão cardiorrespiratória de estudantes da EJA. Essa proposta pode servir como base para que professores tenham meios para desenvolver e avaliar intervenções relacionadas com aptidão física e saúde na EJA.

Palavras-Chave: Aptidão Física; Atividade Física; Esportes; Estudos de Intervenção; IMC.

\section{Introdução}

Estudos têm demonstrado o papel relevante dos níveis adequados de aptidão física para a promoção da saúde ${ }^{1-4}$. O componente da aptidão física mais preponderante parece ser a aptidão cardiorrespiratória (APCR), que quando em baixos níveis, se torna um importante indicador de risco à saúde ${ }^{5,6}$. Desse modo, quando os níveis de aptidão física e atividade física estão baixos e há presença de sobrepeso/obesidade, parece que existe maior predisposição para o desenvolvimento de diabetes tipo 2, doença coronariana, hipertensão arterial, colesterol elevado, entre outras ${ }^{4,7-9}$.

Adicionalmente, já é sabido que a ocorrência de baixos níveis de aptidão física é extensível a toda a população ${ }^{10}$. O número de jovens com sobrepeso, obesidade e risco cardiometabólico tem se tornado um emergente problema de saúde pública ${ }^{11}$. Aproximadamente $50 \%$ e $30 \%$ dos escolares brasileiros apresentam, respectivamente risco à saúde cardiometabólica, sobrepeso e obesidade ${ }^{12}$. No entanto, por ser na escola, e mais especificamente nas aulas de educação física escolar o local de acesso à prática de atividade física da maioria dos jovens brasileiros, tem se tornado crescente a preocupação em organizar as aulas de educação física voltadas ao ensino e a vivência das diferentes manifestaçóes da cultura corporal do movimento associadas à promoção da saúde, considerando as 
especificidades dos diferentes níveis de ensino e o contexto escolar ${ }^{13-16}$.

Dentre os níveis de escolaridade, a educação noturna para jovens e adultos (EJA) merece atenção, principalmente ao considerarmos dentre outros aspectos, a diversidade dos escolares, tanto numa perspectiva social e cultural como numa perspectiva de idade ${ }^{17}$. Mais desafiador ainda será encontrarmos estudos que se preocupem em entender à educação física para esses jovens e adultos. Além disso, parecenos possível enquadrar também nessa reflexão, a escassez de pesquisas que tragam informações especificas sobre o papel da educação física escolar para os jovens adultos. As informaçóes parecem de fato ser restritas, e poucos estudos têm se preocupado em descrever as aulas de educação física, e em criar planejamentos que sejam capazes de envolver os jovens adultos em suas aulas ${ }^{18}$.

\section{Método}

O presente estudo é caracterizado como uma pesquisa avaliativa somativa com pré e pós teste de grupo único em forma de estudo de caso. Foi realizado em uma escola da região carbonífera do Rio Grande do Sul. No município onde foi realizada a pesquisa existem 4 escolas que desenvolvem a EJA totalizando 910 estudantes, de 2032 matrículas divididas em ensino fundamental, médio e EJA. Duas dessas escolas se encontram no mesmo bairro onde há uma instituição de ensino prisional (dentro de um complexo penal) e uma escola de ensino fundamental regular situada a uma quadra fora do complexo. A população total de estudantes nesse bairro era de aproximadamente 732 estudantes, sendo 499 matrículas na EJA na instituição de ensino prisional e as outras na escola de ensino fundamental regular, informação conforme senso escolar de $2014^{24}$.

Por conveniência foram avaliados inicialmente 43 sujeitos de uma população de 74 matriculados na escola de ensino fundamental regular na EJA, devido à facilidade de acesso para pesquisa na escola ser em conjunto com o projeto da Educação Física escolar na instituição. O critério de inclusão adotado para compor a amostra era de no mínimo 75\% de presença nas aulas práticas de Educação Física. Dos sujeitos avaliados inicialmente somente 8 casos cumpriram critério de inclusão e assinaram um termo de consentimento livre e esclarecido,
A partir disso, torna-se relevante um maior número de pesquisas na EFI da EJA, que abordem diferentes perspectivas ${ }^{18-20}$, tais como o planejamento e a metodologia utilizada durante as aulas, o efeito das aulas de educação física sobre indicadores de saúde, o índice de massa corporal e os níveis de aptidão física cardiorrespiratória. Além de que, essa perspectiva se justifica por ofertar aumento dos níveis baixos de atividade física ${ }^{21}$, assim como a possiblidade dessas aulas se tornarem um local de identificação das manifestaçóes da cultura corporal que sejam agradáveis, favorecendo ao engajamento no esporte e na atividade física na EJA ${ }^{22,23}$. Nesse sentindo, o objetivo do presente estudo foi verificar o efeito das aulas de educação física escolar (EFI) nos níveis de aptidão cardiorrespiratória e no índice de massa corporal (IMC) em estudantes na EJA.

que também foi assinado pelos pais dos menores de 18 anos. O presente estudo foi elaborado a partir da aprovação do comitê de ética da Universidade Federal do Rio Grande do Sul, sob o parecer do projeto no 259.883 e em consonância com o disposto na versão atualizada da Declaração de Helsinkii ${ }^{25}$.

A educação física da EJA foi composta por 60 aulas em um semestre, realizadas 3 vezes por semana, durante 20 semanas, de março a julho de 2015. Em 40 dos períodos, foram realizadas aulas práticas concentradas no ensino de habilidades motoras e aumento de movimentação corporal. Com o objetivo de realizar a manutenção das capacidades físicas e da saúde através da prática de atividade física organizada. As outras 20 intervenções foram realizadas de forma teórico-prática. Os conteúdos abordados ao longo do semestre foram: A importância da educação física para a saúde; o ensino do badminton e da ginástica (aulas de jump); noçóes práticas e teóricas sobre alimentação adequada, acompanhamento da pressão arterial e monitoramento da frequência cardíaca. As aulas de educação física foram articuladas ao projeto político pedagógico da escola, inseridas nesse formato a partir de março de 2015 junto a mesma perspectiva do tema de trabalho em vigor na instituição "De todo o jeito se aprende".

Os planos de aulas básicos eram formulados da seguinte maneira: 5 a 15 minutos de aquecimento 
e formação corporal; 10 a 15 minutos de atividade principal; 10 a 15 minutos atividade final: alongamento, explanação sobre hábitos de vida saudáveis e/ou volta à calma. A partir dessas considerações iniciais, o primeiro conteúdo ensinado foi o badminton, por conta da leveza das raquetes e por ser um esporte sem contato físico entre alunos. Entre março e maio, tínhamos como objetivo promover a inclusão de todos os alunos na aula de EFI. De modo que a intervenção náo gerasse esforço físico demasiado para os menos aptos, bem como contemplasse o sentido de desafio para os mais aptos. As aulas eram realizadas sempre com uma estrutura dividida em atividades de aquecimento junto a uma explicação teórica sobre a utilização e objetivo daquela prática para a saúde e para a vida diária, seguida de atividades que abordassem a técnica do esporte e, por fim, o jogo propriamente dito com formas variadas, priorizando a maior participaçáo dos alunos e grande movimentação corporal. O conteúdo de aulas no inverno, foi o ensino do ritmo e expressão corporal através do Jump, o qual foi contextualizado para a escola e consistiu na combinação de saltos, movimentos coreografados em mini trampolins a partir do ritmo de músicas escolhidas em conjunto com os alunos, proporcionando um alto engajamento e conhecimento referente à expressão corporal. Visto que, depois de determinado nível de conhecimento e melhoria da resistência física, os próprios alunos determinavam os movimentos a serem desenvolvidos nas aulas. Essa modalidade foi escolhida devido a escola ter material disponível e os alunos terem curiosidade e interesse. No QUADRO 1 podemos observar as etapas do modelo de intervenção realizado.

QUADRO 1 - Resumo dos métodos de ensino na Educação Física Escolar na EJA.

\begin{tabular}{|l|}
\hline \multicolumn{1}{|c|}{ Início do semestre: } \\
\hline - Entrega do projeto para a direção escolar; \\
- Avaliação das preferências por atividade física; \\
- Apresentação de um esporte diferenciado para os alunos (badminton); \\
- Anamnese relacionada à saúde; \\
- Apresentação do Jump; \\
- Justificava da importância de aulas práticas para a saúde; \\
- Avaliação física relacionada à saúde. (Aptidão Física PROESP-Br). \\
\hline \multicolumn{1}{c|}{ Pontos chave da intervençáo: } \\
\hline - Iniciada pela sustentação teórica de aulas práticas para melhoria da saúde; \\
- Contextualizaçáo dos esportes e da atividade física com a realidade da escola; \\
- Abordagem sobre o conhecimento referente às aulas práticas e sua utilidade no dia-a-dia; \\
- Priorizaçáo de aulas práticas no maior número de momentos possíveis no semestre; \\
- Respeito às capacidades de cada sujeito; \\
- Realizaçáo de aulas práticas de ginástica, no auditório da escola, mesmo em dias de chuva; \\
- Ensino da teoria sobre a intensidade e o controle do corpo durante o exercício físico relacionada com a prática de Jump e \\
Badminton; \\
- Avaliaçóes formativas e feedbacks constantes para e com os alunos; \\
- Alto engajamento e vínculo da educação física com a proposta da escola, os alunos e a cultura escolar; \\
- Flexibilidade do planejamento e reconstruçáo ao longo da intervenção. \\
\hline
\end{tabular}

Para a caracterização dos sujeitos utilizou-se um questionário com perguntas abertas, onde os alunos foram orientados a relatar os problemas que possuíam e que pudessem influenciar na sua frequência nas aulas, bem como, na prática de exercício físico. Essa avaliação também ocorreu de forma informal através de conversas em grupo e individuais registradas nas cadernetas da escola e diário de campo do professor.

A APCR foi avaliada a partir do teste de caminhada/corrida de 6 minutos para escolares proposto por PROESP-Br $r^{4}$. O teste foi realizado na quadra da escola, os instrumentos utilizados para sua realização foram cones, fita métrica e cronômetro. Para medir a estatura foi utilizada uma fita métrica marca Cescorf de precisão de $0,01 \mathrm{~cm}$ estendida de 
baixo para cima e fixada na parede, a medida da massa corporal foi realizada com uma balança de precisão de 100 gramas marca e modelo QF-2003B. O IMC foi mensurado a partir da divisão da massa corporal em quilogramas pela estatura elevada ao quadrado.

O modelo estatístico de equaçóes generalizadas (GEE) para dados não paramétricos, com transformação de parâmetros das matrizes em escala $\log$ link, foi utilizado a fim de verificar o efeito das aulas de EFI escolar sobre o IMC e a APCR dos alunos, estimando intervalos de confiança de 95\% de probabilidade, para um bootstraping de 1000 testes na amostra. $O$ delta de variação em percentual individual e médio foi calculado para verificar o efeito de alteraçáo dos valores de APCR e IMC entre os momentos pré e pós intervenção individualmente. Todos os cálculos foram realizados no software SPSS for Windows versão 20.0 e as figuras construídas no software graphpad prism versão 5.0.

\section{Resultados}

\section{Perfil dos alunos}

O QUADRO 2 apresenta a descrição do perfil de estudantes. Observa-se que o perfil dos sujeitos avaliados apresentou grande variabilidade na faixa etária, nos aspectos de saúde, nos sociais, culturais e escolares o que os caracterizou como uma população especial e heterogênea, com diversas ocupaçôes e funçôes familiares.

\section{Aptidáo cardiorrespiratória e índice de massa corporal}

A TABELA 1 apresenta o efeito das aulas de educação física na APCR e IMC no final do semestre letivo através dos deltas de variaçáo e dos intervalos de confiança. A APCR foi menor no préteste, comparativamente ao pós-teste apresentando um efeito de aumento médio de 59,88 metros o qual demonstrou variabilidade em um intervalo de confiança de $95 \%$ entre 17 a 102 metros de melhora na corrida de 6 minutos. Quanto ao IMC não observamos diferenças relevantes nos valores observados na TABELA 1.

A FIGURA 1 apresenta a variação da APCR em cada sujeito, do pré-teste para o pós-teste. A partir dessa análise individual foi possível perceber que houve melhora da APCR em todos os sujeitos. Sendo que, os que mais aumentaram foram o cinco e o oito, $19,3 \%$ e $20,3 \%$ respectivamente, o que representa que a proposta de EFI foi eficiente para o aumento da APCR ou ao menos proporcionou a manutenção desse parâmetro.

A FIGURA 2 apresenta a variação do IMC em cada sujeito, do pré-teste para o pós-teste. A partir dessa análise individual foi possível perceber que houve uma redução muito pequena do IMC em cinco dos oito sujeitos. Sendo que no sujeito número oito houve redução de $8,3 \%$ na variável, resultados os quais indicam alterações consistentes em apenas alguns dos sujeitos.

QUADRO 2 - Descrição do perfil dos estudantes da EJA.

\begin{tabular}{|c|c|c|c|c|c|c|}
\hline & Sexo & Idade & Ocupaçáo & Perfil relacionado à saúde & $\begin{array}{c}\text { Perfil social/cultural/ } \\
\text { escolar }\end{array}$ & FQ \\
\hline 1 & $\mathrm{~F}$ & 22 & Garçonete & $\begin{array}{l}\text { Hipertensão arterial; utilizava } \\
\text { medicamento controlado; } \\
\text { obesidade em grau elevado. }\end{array}$ & $\begin{array}{l}\text { Participa de atividades } \\
\text { religiosas; possui prole; } \\
\text { dificuldades financeiras e } \\
\text { conflitos familiares. }\end{array}$ & $78,3 \%$ \\
\hline 2 & $\mathrm{~F}$ & 18 & Doméstica & $\begin{array}{l}\text { Não apresenta sintomas rela- } \\
\text { cionados à doenças específicas; } \\
\text { Queixa-se de fortes dores nas } \\
\text { pernas durante o sono. }\end{array}$ & Possui prole. & $80 \%$ \\
\hline 3 & M & 16 & Estudante & Bronquite asmática. & $\begin{array}{c}\text { Apresenta dificuldades } \\
\text { cognitivas. }\end{array}$ & $83,3 \%$ \\
\hline
\end{tabular}

continua 
continuação

\begin{tabular}{|c|c|c|c|c|c|c|}
\hline & Sexo & Idade & Ocupação & Perfil relacionado à saúde & $\begin{array}{c}\text { Perfil social/cultural/ } \\
\text { escolar }\end{array}$ & FQ \\
\hline 4 & M & 45 & $\begin{array}{c}\text { Pintor } \\
\text { Residencial }\end{array}$ & $\begin{array}{l}\text { Diabetes tipo II e hipertensão } \\
\text { arterial. Utiliza medicamento } \\
\text { controlado. Problemas } \\
\text { osteomusculares na coluna e } \\
\text { membros superiores. }\end{array}$ & $\begin{array}{l}\text { Dificuldades de comunica- } \\
\text { çáo e cognitivas. }\end{array}$ & $96,6 \%$ \\
\hline 5 & M & 17 & $\begin{array}{l}\text { Cuidador } \\
\text { de equinos }\end{array}$ & $\begin{array}{c}\text { Estudante sofreu ferimento } \\
\text { há } 3 \text { anos de arma branca na } \\
\text { regiáo do abdômen. Sofre com } \\
\text { dores na região. }\end{array}$ & $\begin{array}{l}\text { Alto interesse por esportes. } \\
\text { Dificuldades financeiras e } \\
\text { conflitos familiares. }\end{array}$ & $91,6 \%$ \\
\hline 6 & $\mathrm{~F}$ & 52 & $\begin{array}{l}\text { Dona de } \\
\text { casa }\end{array}$ & $\begin{array}{c}\text { Hipertensão arterial; usa } \\
\text { medicamento controlado; } \\
\text { realizou uma cirurgia na } \\
\text { região da coluna lombar com } \\
4 \text { parafusos e uma chapa em } \\
\text { titânio. }\end{array}$ & $\begin{array}{c}\text { Estudante proativa; } \\
\text { Religiosa e solidária. Possui } \\
\text { prole. }\end{array}$ & $100 \%$ \\
\hline 7 & $\mathrm{~F}$ & 40 & Cozinheira & $\begin{array}{c}\text { Sofre de fortes dores nas costas, } \\
\text { dificuldade motora e restriçáo } \\
\text { de movimentos nos braços. } \\
\text { Diabetes tipo II. }\end{array}$ & $\begin{array}{l}\text { Possui prole com necessi- } \\
\text { dades especiais. }\end{array}$ & $96,6 \%$ \\
\hline 8 & M & 26 & $\begin{array}{l}\text { Aposentado } \\
\text { por defici- } \\
\text { ência }\end{array}$ & $\begin{array}{c}\text { Traços de autismo, } \\
\text { esquizofrenia e outros } \\
\text { distúrbios comportamentais. } \\
\text { Utiliza medicaçáo controlada } \\
\text { diariamente, inclusive } \\
\text { na escola. Não apresenta } \\
\text { diagnóstico conclusivo sobre } \\
\text { sua deficiência. }\end{array}$ & $\begin{array}{l}\text { O aluno foi transferido da } \\
\text { APAE para a escola. Alta } \\
\text { capacidade de memoriza- } \\
\text { ção de números e informa- } \\
\text { çôes sistematizadas. }\end{array}$ & $100 \%$ \\
\hline
\end{tabular}

TABELA 1 - Falta título.

\begin{tabular}{cccccc}
\hline $\begin{array}{c}\text { Médias } \\
(\text { GEE })\end{array}$ & $\begin{array}{c}\text { Pré-teste } \\
(\text { IC95\% })\end{array}$ & $\begin{array}{c}\text { Pós-teste } \\
(\text { IC95\% })\end{array}$ & Efeito médio & $\mathbf{X}^{2}$ & $\mathbf{p}$ \\
\hline APCR & $\begin{array}{c}675,25 \\
(565,88 \text { a } 784,62)\end{array}$ & $\begin{array}{c}735,13 \\
(594,22 \text { a } 876,03)\end{array}$ & $\begin{array}{c}+59,88 \\
(17,2 \text { a } 102,55)\end{array}$ & 7,56 & $0,006^{*}$ \\
IMC & 32,75 & 32,02 & $-0,72$ & 1,78 & 0,181 \\
\hline
\end{tabular}

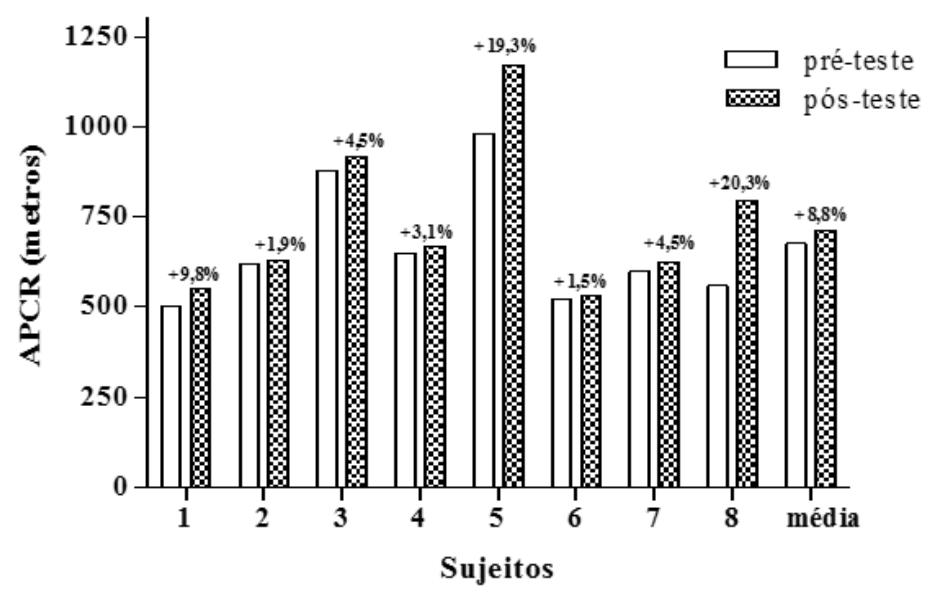

FIGURA 1 - Variação da aptidão física cardiorrespiratória em cada sujeito e na média do grupo. 


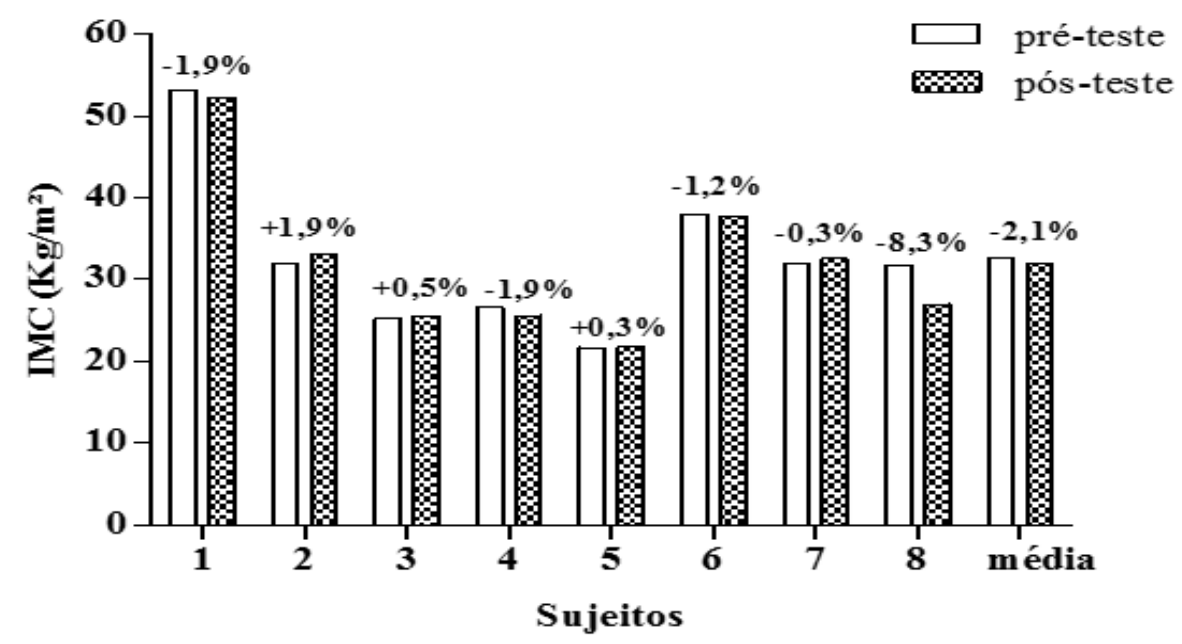

FIGURA 2 -Variação do IMC em cada sujeito e na média do grupo.

\section{Discussão}

O objetivo do presente estudo foi verificar o efeito das aulas de educação física escolar nos níveis de aptidão cardiorrespiratória e no índice de massa corporal (IMC) em estudantes na EJA. Nossos resultados evidenciam um aumento significativo nos níveis de aptidão cardiorrespiratória. Por outro lado, as alteraçóes no IMC foram reduzidas e inconsistentes. No entanto, ressaltamos que mesmo sob a presença de obesidade, diabetes tipo II e traços de autismo, houve um acréscimo importante de até $20,3 \%$ na APCR e 8,3\% de redução no IMC em alguns dos estudantes que participaram de $75 \%$ das aulas de EFI, apontando para o efeito benéfico do exercício físico na saúde cardiometabólica dos estudantes. Esses resultados foram muito relevantes diante da perspectiva pedagógica, porque indicam melhorias em indicadores de saúde de pessoas que necessitavam de uma intervenção, e foram muito expressivos considerando as vulnerabilidades e dificuldades para docentes e alunos darem efetividade às aulas na $\mathrm{EJA}^{22,23}$.

Nesse mesmo sentido, estudos indicam que aulas de educação física planejadas e estruturadas com métodos semelhantes aos nossos apresentam efeitos que variam de 1 a $15 \%$ no que se refere ao aumento da aptidão física cardiorrespiratória ${ }^{26,27}$, estando de acordo com os efeitos do presente estudo (8,8\% FIGURA 1). Destacamos que embora pequeno, o aumento da APCR na EJA foi muito relevante aos sujeitos devido a esse ser considerado como um importante indicador de saúde ${ }^{28}$, desde a infância até a vida adulta, na medida em que há associação inversa entre essa variável e mortalidade por doenças cardiovasculares ${ }^{29}$. Além disso, o risco de morte parece ser mais dependente do nível de APCR, comparativamente ao IMC, no que se refere a proteção da saúde cardiovascular e metabólica ${ }^{30}$. Por outro lado, estudos corroboram com a dificuldade em se obter efeitos sobre o IMC apenas através da prática de atividade física e exercício ${ }^{31,32}$. A esse respeito se sabe que é necessária uma intervenção multidisciplinar ou orientação nutricional ${ }^{32}$, com um período de tempo maior ao do presente estudo, para assim obter efeitos relevantes sobre a variável. Ademais, entende-se que esses são fatos bastante difíceis de se realizar nas escolas públicas brasileiras.

A partir dos presentes achados, acreditamos que é papel da educação física escolar, seja para crianças ou jovens e adultos, fazer com que os alunos compreendam e obtenham os benefícios da prática regular da atividade física e da melhora da aptidão física para sua saúde ${ }^{33}$. Para tanto, a EFI deve despertar nos alunos o prazer e o gosto pelo exercício físico e pela prática dos diferentes esportes, sendo que as diversas características sociais e culturais dos alunos da EJA devem ser respeitadas ${ }^{18-20,22,23}$. De fato, na presente pesquisa observamos pessoas com diversos tipos de emprego e funções sociais distintas, sendo 
trabalhadores em sua maioria. Salientamos ainda que devido à idade avançada dos alunos da EJA, alguns dos sujeitos já são portadores de doenças inerentes à síndrome metabólica como a hipertensão arterial e o diabetes mellitus tipo II (QUADRO 2). Nesse sentido, foi essencial a utilização de um questionário de anamnese e uma avaliação física antes da intervenção com exercícios físicos na EJA. Bem como, durante as aulas, as intensidades dos exercícios foram controladas observacionalmente para que riscos à saúde como uma pressão arterial muito alta, hiperglicemia ou hipoglicemia dos alunos diabéticos fosse evitada ${ }^{34}$. Esses são fatos que explicam a dificuldade de se aumentar os níveis de aptidão física para escolares da EJA, justificando os limitados efeitos encontrados em alguns sujeitos do estudo.

Como limitaçóes para a presente pesquisa, destacamos o pequeno número de sujeitos que pode ser explicado pela alta evasão de estudantes, visto que a prática em EFI não é obrigatória para todos, segundo alguns critérios impostos pela legislação ${ }^{35}$. Salientamos também que não é possível realizar generalizaçóes pois os sujeitos foram selecionados de modo conveniente. Não avaliamos objetivamente a percepção sobre o significado de saúde para os sujeitos da EJA envolvidos na pesquisa, embora tenhamos observado, que os mesmos percebiam certa melhora em alguns outros parâmetros além dos avaliados, como a auto estima, auto eficácia e a imagem de si mesmo, o que se torna uma hipótese relevante a ser testada em futuras pesquisas. Também não foram controlados possíveis fatores intervenientes como: atividade física fora da escola e nem os padrôes alimentares. Sugerimos que sejam realizados futuros estudos que indiquem pontos de corte relacionando medidas da APCR no teste de corrida/caminhada de 6 minutos $^{4}$ para adultos, acima dos 17 anos com marcadores fisiológicos de risco à saúde como glicemia, colesterol, pressáo arterial, triglicerídeos etc. No entanto, cabe ressaltar que apesar das limitaçóes, esse é um dos poucos estudos que trata do tema da importância das aulas de educação física escolar na perspectiva da promoção de indicadores de saúde de jovens e adultos. Sugerindo a importância das aulas de EFI, na perspectiva de promover níveis de aptidão física adequados nessa população, assim como, um desenho de pesquisa adequado para que sejam evidenciados os benefícios das aulas de EFI.

Dessa forma, concluímos que o programa de educação física escolar utilizado no presente estudo contribuiu para melhora dos níveis de aptidão cardiorrespiratória de estudantes da EJA. Portanto, indicamos que é possível através da educação física escolar aumentar a proteção à saúde para as possíveis doenças cardiometabólicas dessa população. Além desse importante resultado, o presente estudo pode servir como uma base para que professores de EFI possam efetivamente desenvolver e avaliar intervenções na EJA de modo simples e prático.

\begin{abstract}
Effects of physical education in levels of cardiorespiratory fitness and body mass index in youth and adults education- YAE

The aim of present study was to verify the effect of physical education lessons at school (PE) in levels of cardiorespiratory fitness and body mass index (BMI) in students of the Youth and Adults Education (YAE). It was a summative evaluation research carried out in a school of RS, it elaborated with 8 subjects which, between 16 and 52 years old to both sex, from a total population of 74 students, that fulfilled $75 \%$ of presence in SPE lessons on the YAE. Cardiorespiratory fitness (CR) and body mass index (BMI) were assessed by PROESP-Br battery tests at the beginning of the first semester of 2015 and in the end of this period. The PE effects in the group were measured from the statistical model of generalized estimative equations (GEE) in 95\% confidence intervals $(95 \% \mathrm{Cl})$ and delta mean percentage. We also described the percentage of variation of $\mathrm{CR}$ and $\mathrm{BMI}$ for each subject. Thus, it was observed that the $\mathrm{CR}$ increased significantly from pre-test ( 675.25 meters) to the post-test $(735.13$ meters), $(95 \% \mathrm{Cl}=+17.2$ to +102.55$)$, which corresponds to an average increase of $8.8 \%,(p=0.006)$. All subjects increased APCR in the individual assessment,
\end{abstract}


these results were not observed for BMI. We conclude that the physical education program contributed in improving cardiopulmonary fitness levels of students in adult education. This proposal can be used as a basis for which teachers have a means for developing and accessing interventions related to physical fitness and health in YAE.

KeYwords: Physical Fitness; Physical Activity; Sports; Intervention Studies; BMI.

\section{Referências}

1. Blair SN, Cheng Y, Holder JS. Is physical activity or physical fitness more important in defining health benefits? Med Sci Sports Exerc. 2001;33(6):379-399.

2. Lee D, Artero EG, Sui X, Blair SN. Mortality trends in the general population: the importance of cardiorespiratory fitness. J Psychopharmacol. 2010;24(4):27-35.

3. Glaner MF. Importância da aptidão física relacionada à saúde. Rev Bras Cineantropom Desempenho Hum. 2003;5(2):75-85.

4. Gaya ACA, Gaya AR. PROESP-Br Manual de testes e avaliação. Porto Alegre: Editora Perfil UFRGS; 2016.

5. Elliot E, Erwin H, Hall T, Heidom B. Comprehensive School Physical Activity Programs: Helping All Students Achieve 60 Minutes of Physical Activity Each Day. JOPERD [Internet]. 2013[citado__];84(9):9-15. Disponível em: http:// www.shapeamerica.org/advocacy/positionstatements/pa/upload/CSPAP-Position-Statement-2.pdf.

6. Ortega FB, Ruiz JR, Castillo MJ, Sjöström M. Physical fitness in childhood and adolescence: a powerful marker of health. Int J Obes. 2008;32(1):1-11.

7. Gander JC, Sui X, Hébert JR, Hazlett LJ, Cai B, Lavie CJ, et al. Association of Cardiorespiratory Fitness With Coronary Heart Disease in Asymptomatic Men. Mayo Clin Proc. 2015;90(10):1372-1379.

8. Gaya AR, Alves A, Aires L, Martins CL, Ribeiro JC, Mota J. Association between time spent in sedentary, moderate to vigorous physical activity, body mass index, cardiorespiratory fitness and blood pressure. Ann Hum Biol. Taylor $\&$ Francis. 2009;36(4):379-387.

9. Andaki ACR, Tinoco ALA, Andaki R, Santos A, Brito CJ, Mendes EL. Nível de atividade física como preditor de fatores de risco cardiovasculares em crianças. Motriz Rev Educ Fis. 2013;19(3):8-15.

10. Corseuil MW, Petroski EL. Baixos níveis de aptidão física relacionada à saúde em universitários. Rev Bras Educ Fís Esporte. 2010;24:49-54.

11. World Health Organization. Health Topics Obesity [Internet]. Genebra: WHO; 2015 [citado_ ]. p.1. Disponível em: http://www.who.int/topics/obesity/en/.

12. Flores LS, Gaya AR, Petersen RDS, Gaya A. Trends of underweight, overweight, and obesity in Brazilian children and adolescents. J Pediatr. 2013;89(5):456-61.

13. de Barros MVG, Nahas MV, Hallal PC, de Farias Júnior JC, Florindo AA, Honda de Barros SS. Effectiveness of a school-based intervention on physical activity for high school students in Brazil: the Saude na Boa project. J Phys Act Health. 2009;6(2):163-9.

14. Moreira RB. Níveis de atividade física nas aulas de educação física. Porto Alegre (RS): Universidade Federal do Rio Grande do Sul, Escola de Educação Física; 2014.

15. Oliveira LCV. Análise dos efeitos de um programa de educação física relacionado à promoção da saúde sobre a aptidão física de escolares. Porto Alegre (RS): Universidade Federal Do Rio Grande do Sul, Escola de Educação Física; 2014.

16. Denti J, Vargas Â. A influência da dança de rua no nível de ansiedade das mulheres que frequentam o Ensino de Jovens e Adultos - EJA da Escola Estadual Cardeal Roncalli - Frederico Westphalen - RS- Brasil. FIEP Bull. 2013;83:628-30.

17. Silva KS, Lima D da S, Madeira MO. A educação física sob a ótica dos alunos da educação de jovens e adultos no municipio de rio pomba-mg. FIEP Bull. 2013;83.

18. Rodrigues ALM, Bastos APP, Rossato MJV. Physical Education in Youngster and Adult Education. FIEP Bull. 2008;78:169-72.

19. Machado MM. Formação de professores para EJA. Rev Retratos da Esc. 2008;2(2-3):161-74.

20. Sudbrack AP. Práticas e conceitos da Educação Física na Educação de Jovens e Adultos: Uma intervenção baseada na relação entre professor/estudante e nas ferramentas necessárias para uma maior adesão às aulas. Porto Alegre (RS): 
Universidade Federal do Rio Grande do Sul, Escola de Educação Física; 2013.

21. Sallis JF, Bull F, Guthold R, Heath GW, Inoue S, Kelly P, et al. Series Physical Activity 2016 : Progress and Challenges Progress in physical activity over the Olympic quadrennium. Lancet. 2016;6736(16):1-12.

22. Carvalho RM. Educação Física na Educação de Jovens e Adultos. Rev Lugares Educ. 2013;3(5):37-49.

23. Balestrin G. Planejamento em Educaçáo Física : Um Estudo de Caso na Educação de Jovens e Adultos ( EJA ). Ijuí (RS): Universidade Regional Do Noroeste Do Estado do Rio Grande do Sul; 2015.

24. Secretaria Estadual de Educação do Rio Grande do Sul. Censo Escolar 2014 - Dados Finais [Internet]. Porto Alegre: SEDUC-RS; 2015 [citado ]. Disponível em: http://www.educacao.rs.gov.br/pse/html/estatisticas. jsp?ACAO=acao 1 .

25. Association WM. World Medical Association Declaration of Helsinki: Ethical Principles for Medical Research Involving Human Subjects (1964). JAMA. 2013;310:1-5.

26. Hayes RM, Thompson LM, Gress T, Cottrell L, Pino I, Gilkerson CL, et al. Effects of a Brief Physical Activity Program on Young Students' Physical Fitness. Clin Pediatr (Phila). 2015;54(12):1145-52.

27. Minatto G, Barbosa Filho VC, Berria J, Petroski EL. School-Based Interventions to Improve Cardiorespiratory Fitness in Adolescents: Systematic Review with Meta-analysis. Sport Med. 2016;46(9):1273-92.

28. Ortega FB, Ruiz JR, Castillo MJ, Sjöström M. Physical fitness in childhood and adolescence: a powerful marker of health. Int J Obes. 2008;32(1):1-11.

29. Kodama S, Saito K, Tanaka S. Cardiorespiratory Fitness as a Quantitative predictor of all cause mortality and cardiovascular events in healthy men and women: a meta-analysis. JAMA. 2009;301(19):2024-2035.

30. Barry VW, Baruth M, Beets MW, Durstine JL, Liu J, Blair SN. Fitness vs. Fatness on All-Cause Mortality: A MetaAnalysis. Prog Cardiovasc Dis. 2014;56(4):382-390.

31. Santanasto AJ, Newman AB, Strotmeyer ES, Boudreau RM, Goodpaster BH, Glynn NW. Effects of Changes in Regional Body Composition on Physical Function in Older Adults: A Pilot Randomized Controlled Trial. J Nutr Health Aging. 2015;19(9):913-21.

32. Espíndola JA, Capistrano R, Alexandre JM, Silva J Da, Beltrame TS. Efeitos do exercício físico na aptidão física de crianças com sobrepeso. ConScientiae Saúde. 2013;13(2):281-8.

33. Oliveira LCV de, Braga FCC, Lemes VB, Dias AF, Brand C, Mello JB, et al. Effect of an intervention in Physical Education classes on health related levels of physical fitness in youth. Rev Bras Atividade Física Saúde. 2017;22(1):46-53.

34. Mendes R, Sousa N, Reis VM, Barata JLT. Programa de Exercício na Diabetes Tipo 2. Rev Port da Diabetes. 2011;6(2):62-70.

35. Brasil. Lei n. 9394 de 20 dezembro de 1996. Estabelece as diretrizes e bases da educação nacional. Diário Oficial da Uniāo, Brasília (1996 dez. 23); Sec.1:27833.

ENDERECOO DE CORRESPONDÊNCIA: Vanilson Batista Lemes

Rua José Maria de Carvalho, $\mathrm{n}^{\mathrm{o}} 11$ - Bairro Centro Charqueadas - RS - BRASIL CEP: 96745-000 E-mail: vanilson.lemes@hotmail.com; lemesvb@gmail.com
Submetido: 20/01/2016

$1^{\mathrm{a}}$ revisão: $12 / 12 / 2017$

$2^{\text {a }}$ revisão: $11 / 04 / 2018$

Aceito: 07/05/2018 